\title{
Pulmonary botryomycosis: A difficult diagnosis in the preoperative patient
}

\author{
Russell Hodgson, MBBS, Shane A. Blackmore, MBBS, and \\ C. Peter Clarke, FRACS, Melbourne, Australia
}

$\mathrm{W}$ e report a case of primary pulmonary botryomycosis that is the first in English literature to be investigated with positron emission tomography (PET) scanning before operation. This is a rare chronic bacterial infection of the pulmonary system that is difficult to distinguish from bronchial carcinoma before operation.

\section{Clinical Summary}

We present the case of a 62-year-old woman from outback Victoria, Australia, who presented to her local doctor with left-sided chest wall pain. Her chest x-ray film demonstrated a large lesion in the left upper lobe. She had a history of migraines and mild osteoarthritis and had quit smoking 10 months earlier after a 15 pack per year habit. She was further investigated with a computed tomography (CT) scan, which demonstrated a large lesion in the left upper lobe adjacent to the pleura, and a PET scan (Figure 1). The PET scan confirmed high fluorine-18 deoxyglucose (FDG) uptake in the mass with equivocal uptake in the hilar nodal tissue. A tissue diagnosis was attempted by bronchoscopy and washings, and a CT-guided percutaneous needle biopsy. Both attempts failed to find any neoplastic tissue. The biopsy revealed no granulomata. Sputum revealed polymorphs and some nonspecific gram-positive cocci.

She underwent an open thoracotomy and a left upper lobectomy. The mass was adherent to the chest wall, and the offending section of pleura was also stripped. Some soft enlarged hilar lymph nodes were identified and resected with the specimen. The pleura was examined as a frozen specimen, and no neoplastic cells were identified. The lung pathology demonstrated multifocal areas of suppuration and tissue necrosis containing granules of gram-positive cocci surrounded by a fringe of eosinophilic material (Splendore-Hoeppli phenomenon), and a diagnosis of primary pulmonary botryomycosis was made. An opinion was sought from our infectious disease unit, and the decision was not to treat with long-term antibiotics. The patient fully recovered.

\footnotetext{
From the Department of Surgery, Austin Health, Heidelberg, Victoria Australia.

Received for publication May 11, 2005; accepted for publication June 7, 2005.

Address for reprints: Russell Hodgson, MBBS, Department of Surgery, Austin Health, Studley Rd, Heidelberg, Victoria, 3084, Australia (E-mail: russell.hodgson@gmail.com).

J Thorac Cardiovasc Surg 2005;130:924-5

$0022-5223 / \$ 30.00$

Copyright $\odot 2005$ by The American Association for Thoracic Surgery

doi:10.1016/j.jtcvs.2005.06.001
}

\section{Discussion \\ Primary pulmonary botryo- mycosis is a rare condition with only 16 cases de- scribed before $2005 .{ }^{1}$ Bot- ryomycosis was first de-} scribed by Bollinger in 1870 as a disease of horses. ${ }^{1}$ Fungi were thought to the causative factor; however, in 1919 Magrou successfully isolated Staphylococcus aureus from his horse specimen and reproduced the disease in guinea pigs. ${ }^{2}$ Since that time, other microbes such as Pseudomonas aeruginosa, Escherichia coli, alpha-hemolytic streptococcus, Actinobacillus lignieresii, Actinobacillus actinomycetemcomitans, and Serratia marcescens have also been described. ${ }^{1,2}$ Predisposing factors including cystic fibrosis and acquired immune deficiency syndrome have been implicated in many cases, with 11 of the 16 cases of primary pulmonary botryomycosis possibly related to impaired immune responses. ${ }^{1,3,4}$

Pathologically, botryomycosis is characterized by the presence of eosinophilic granules, with eosinophils surrounding central foci of necrosis, known as the Splendore-Hoeppli effect (Figure 2). ${ }^{5}$ The difference between botryomycosis and fungal infection (eg, with actinomyces) is the bacteria shown on Gram staining.

The presentation of patients with pulmonary botryomycosis is varied with symptoms including chronic cough, hemoptysis, and chest wall pain described. ${ }^{1,2,4}$ Clinical examination is usually unremarkable, with the exception of decreased breath sounds over the consolidated lung. ${ }^{4}$ Most previously documented cases were investigated with fine-needle biopsy to obtain a tissue diagnosis before open biopsy. A minority of authors, such as Katapadi and colleagues, ${ }^{3}$ successfully diagnosed botryomycosis on fine-needle biopsy. The diagnosis was not clarified in all documented cases with CT scan, with reports that lesions were highly suspicious for malignancy. The majority of reported cases progressed to open biopsy, usually lobectomy, to exclude malignancy as the primary diagnosis. ${ }^{1,2,4}$

There are no other documented cases of PET scan reports in primary pulmonary botryomycosis. Our scans demonstrated marked uptake in the region of the lung mass, consistent with a diagnosis of malignancy or infection. Similarly, uptake in the hilar region is also consistent with hilar node involvement of malignancy or lymphadenitis.

We submit the need for thorough preoperative workup for lung lesions suspected of being bronchogenic carcinomas. Unfortunately, even with the addition of PET scanning, the majority of patients with pulmonary botryomycosis, a rare chronic infection, will ultimately require open biopsy to exclude malignancy. 


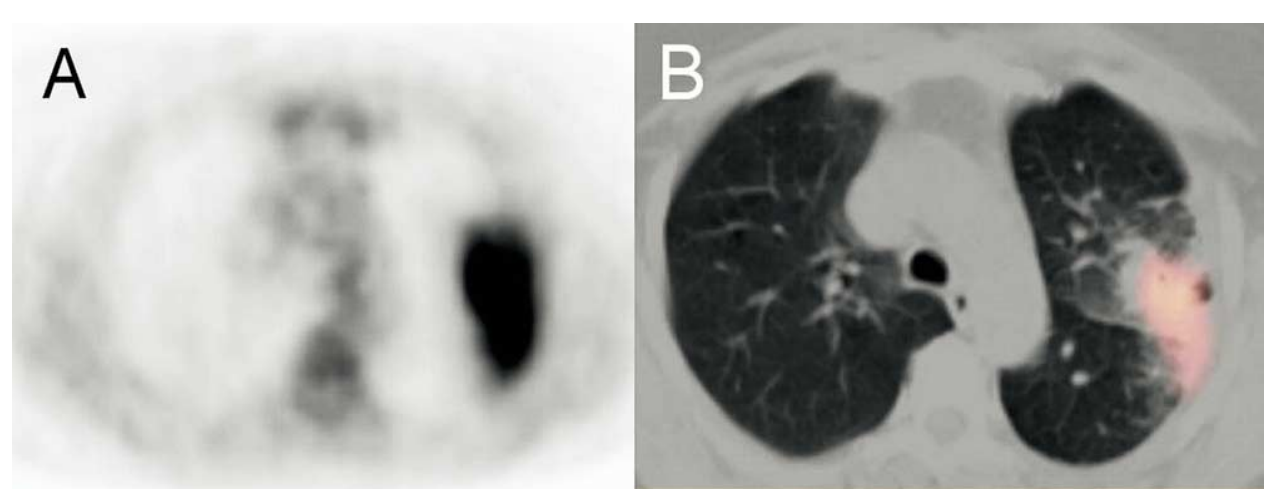

Figure 1. Corresponding positron emission tomography (PET) (A) and computed tomography (CT) with PET overlay (B) images of our patient's left upper lobe mass demonstrating marked fluorine-18 deoxyglucose (FDG) uptake corrolating with the dense mass on CT.

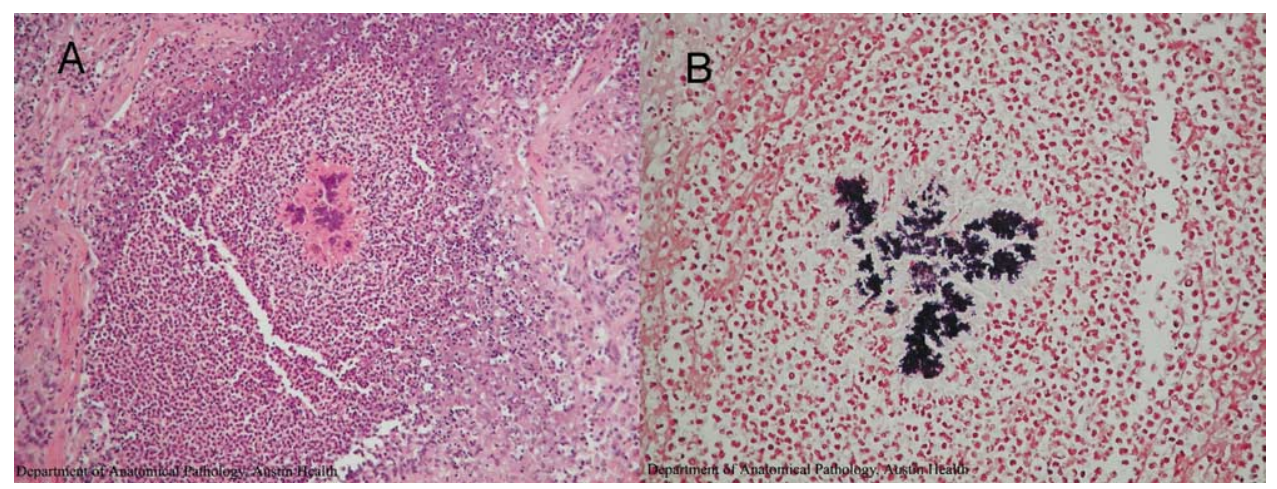

Figure 2. A, Histopathology photos of our patient's lungs demonstrating a microabscess with a surrounding eosinophilic halo (Splendore-Hoeppli effect). B, Gram stain with gram-positive cocci (later shown to be Streptococcus) staining blue.

\section{References}

1. Kathir K, Dennis C. Primary pulmonary botryomycosis: an important differential diagnosis for lung cancer. Respirology. 2001;6:34750.

2. Bersoff-Matcha SJ, Roper CC, Liapis H, Little Jr. Primary pulmonary botryomycosis: case report and review. Clin Infect Dis. 1998;26:620-4
3. Katapadi K, Pujol F, Vuletin JC, Katapadi M, Pachter BR. Pulmonary botryomycosis in a patient with AIDS. Chest. 1996;109:276-7.

4. Paz HL, Little BJ, Ball WC Jr, Winkelstein JA. Primary pulmonary botryomycosis is a manifestation of chronic granulomatous disease. Chest. 1992;101:1160-1.

5. Gavin PJ, Das L, Chadwick EG, Yogev M. Botryomycosis in a child with acquired immunodeficiency syndrome. Pediatr Infect Dis J. 2000; 19:900-1. 